\title{
Remodelamento cirúrgico do coração no tratamento cirúrgico da cardiomiopatia isquêmica
}

\author{
Hemerson GAMA*, William MARTIN*, Surendra K. NAIK*
}

RBCCV 44205-537

Gama H, Martin W, Naik S K - Remodelamento cirúrgico do coração no tratamento cirúrgico da cardiomiopatia isquêmica. Rev Bras Cir Cardiovasc 2001; 16(2): 114-8.

RESUMO: Objetivo: Apresentamos um grupo de pacientes em fase avançada de miocardiopatia isquêmica, operado com a técnica de remodelamento cirúrgico do coração: revascularização do miocárdio, anuloplastia mitral/tricúspide, plicatura de VE e procedimento de DOR.

Casuística e Métodos: Vinte e sete pacientes com miocardiopatia isquêmica avançada foram submetidos a remodelamento cirúrgico do coração: 22 homens, idade média 57,8 anos, 23 em classes III e IV, fração de ejeção média de $15 \%$. Todos os pacientes foram submetidos a CEC e cardioplegia sangüínea quente. Vinte e seis pacientes receberam 74 enxertos (2,7). Anuloplastia mitral foi realizada em 10 casos e mitral + tricúspide em 4. Plicatura do VE foi realizada em 2 pacientes e procedimento de DOR em 18. O tempo médio de pinçamento aórtico foi de $59 \mathrm{~min}$ e o tempo médio de CEC de $145 \mathrm{~min}$. Dez pacientes necessitaram de balão intra-aórtico e 25 receberam drogas inotrópicas no pós-operatório.

Resultados: O tempo médio de ventilação foi de 31,7horas e tempo médio de internação na UTI foi de 65,7 horas. Três pacientes foram reintubados e 2 reoperados por sangramento. Oito pacientes apresentaramom FA e 1 AVC. O período médio de internação foi de 13 dias. A mortalidade hospitalar foi de 22,3\% (6 pacientes); 4 decorrente de baixo débito e 2 de sepse. Num período de acompanhamento médio de 20,7 meses, 19 (70,3\%) estavam vivos; 17 em classes I e II, não tendo sido observado IAM neste período. Dois pacientes foram reinternados por FA, 2 por angina e 3 por ICC.

Conclusão: Remodelamento cirúrgico do coração é uma alternativa cirúrgica para pacientes com cardiomiopatia isquêmica com morbidade aceitável e bons resultados a médio prazo.

DESCRITORES: Procedimentos cirúrgicos cardíacos, métodos. Isquemia miocárdica, cirurgia.

\section{INTRODUÇÃO}

A cardiomiopatia isquêmica apresenta uma alta morbidade e mortalidade. Permanece nos dias de hoje como a causa mais freqüente de óbito. O seu tratamento médico já está bem estabelecido e tem limitações claras. Pacientes com fração de ejeção menor que $20 \%$ apresen- tam uma sobrevida, em dois anos, menor que $25 \%$ (1). Transplante cardíaco apresenta, ainda, os melhores resultados no tratamento cirúrgico da miocardiopatia isquêmica. Todavia, devido à alta demanda de órgãos e ao crônico problema nas doações, iniciou-se a procura de alternativas para o tratamento cirúrgico desta doença.

\footnotetext{
Trabalho realizado no Cardiothoracic Surgery Department, Glasgow Royal Infirmary University NHS Trust. Glasgow, G4, UK. *Do Surgery Department, Glasgow Royal Infirmary University NHS Trust.

Liberado para publicação em março de 2001.

Endereço para correspondência. Hemerson Gama. Rua Coronel Guardea, 11 apto 6. Centro. Cachoeiro do Itapemirim, ES. Brasil. CEP 29030-000. Tel.: (27) 522-7923. e.mail: hgama@usa.net
} 
Gama H, Martin W, Naik S K - Remodelamento cirúrgico do coração no tratamento cirúrgico da cardiomiopatia isquêmica. Rev Bras Cir Cardiovasc 2001; 16(2): 114-8.

O conceito de miocárdio hibernante fez com que a revascularização do miocárdio se tornasse uma alternativa para o transplante cardíaco (1-4) e apesar de um risco elevado, apresenta excelentes resultados quando bem indicada ${ }^{(5-8)}$. Outras alternativas surgiram ao longo dos últimos anos, como a cardimioplastia ${ }^{(9)}$, aortomioplastia, ventrículo de músculo esquelético, revascularização transmiocárdica através de laser ${ }^{(10)}$, dispositivo de assistência ventricular e coração artificial $^{(11)}$ e ainda outras formas de transplante como o xenotransplante ${ }^{(12)}$ e o transplante heterotópico. Recentemente, BATISTA et al.(13) descreveram a técnica de ventriculotomia parcial, que consiste na ressecção de parte do ventrículo esquerdo no sentido de reduzir o stress da parede e restaurar a relação normal entre massa e volume, melhorando assim a função ventricular ${ }^{(14)}$. Com um princípio semelhante, a técnica descrita por Dor, para tratamento de aneurisma de ventrículo esquerdo, que consiste na exclusão da área aneurismática, utilizando-se um retalho de Dacron, a qual é denominada de endoaneurismorrafia(15-17) tem sido utilizado no tratamento da miocardiopatia isquêmica ${ }^{(18)}$. Embora alguns autores tenham observado melhora da regurgitação valvar mitral apenas com revascularização do miocárdio (19) a anuloplastia da valva mitral auxilia a reduzir a sobrecarga de volume do ventrículo esquerdo, melhorando a função ventricular e, portanto, a sintomatologia do paciente (20-21). Adicionalmente, a anuloplastia mitral melhora a geometria do ventrículo esquerdo melhorando a sua função (22).

Apresentamos nossa experiência inicial com o remodelamento cirúrgico do coração no tratamento cirúrgico da cardiomiopatia isquêmica, analisando a indicação, técnica cirúrgica e resultados a curto e médio prazos.

\section{CASUÍSTICA E MÉTODOS}

Dos pacientes referidos ao nosso Serviço com indicação para transplante cardíaco, apenas $1 / 3$ são incluídos na lista de espera. Os pacientes que não preenchem os critérios de seleção para transplante (hipertensão pulmonar severa, doença vascular periférica, etc) são considerados para operação convencional como alternativa ao tratamento clínico. O remodelamento cirúrgico do coração consiste em revascularização completa do miocárdio, sempre que houver áreas de miocárdio viável identificadas pela medicina nuclear e a cineangiocoronariografia demonstrar uma artéria abordável do ponto de vista cirúrgico; anuloplastia mitral e/ou tricúspide na presença de regurgitação importante e correção de aneurisma de ventrículo esquerdo. A operação é planejada de acordo com as informações fornecidas pelo ecocardiograma, no que diz respeito a regurgitação valvar, aparelho subvalvar, espessura de paredes, diâmetro de câmaras, demonstração do aneurisma, etc. Os dados angiográficos, tais quais anatomia das artérias coronárias e seus efeitos distais, ventriculografia, pressões de capilar pulmonar, e pressões diastólicas e sistólicas dos ventrículos, além da demonstração de aneurisma de ventrículo esquerdo.

Além da coronariografia, a revascularização do miocárdio foi indicada baseada no resultado da cintilografia do miocárdio com tálio para definir áreas de miocárdio viável.

Vinte e sete pacientes, portadores de miocardiopatia isquêmica avançada, foram operados com esta técnica no nosso Serviço. Vinte e dois pacientes eram homens e 5 mulheres, com idade média de 57,8 anos (variando de 43-73 anos). A maioria se encontrava em classe funcional III e IV da NYHA. A fração média de ejeção do ventrículo esquerdo, medida através de cintilografia miocárdica com thallium, foi de $15 \%$ (variando de 8 a 23\%).

A operação foi realizada com circulação extracorpórea à temperatura de $28^{\circ} \mathrm{C}$, canulação aórtica e das veias cavas e descompressão do ventrículo esquerdo através da veia pulmonar superior direita. A proteção miocárdica utilizada foi cardioplegia sangüínea normotérmica anterógrada e retrograda, com solução de St Thomas + potássio. Em todos os pacientes foi realizada revascularização do miocárdio + anuloplastia mitral e/ou ventriculoplastia.

Vinte e seis pacientes receberam, ao todo, 74 enxertos, com uma média de 3.1 enxertos por paciente (variando de 1 a 4). Anuloplastia mitral foi efetuada em 14 pacientes e anuloplastia tricúspide concomitante em 4 dos casos. $O$ anel utilizado foi o de Carpantier-Edwards $\AA$ e o tamanho variou de 28 a $32 \mathrm{~mm}$. Dezoito pacientes tiveram o aneurisma corrigido pela técnica de Dor, utilizando-se retalho de Dacron impregnado com colágeno (Vascutek ${ }^{\circledR}$ ) e em 2 a plastia do ventrículo esquerdo foi feita através de plicatura simples. O tempo médio de pinçamento aórtico foi de 59 minutos (variando de $14-100 \mathrm{~min}$ ) e o tempo médio de circulação extracorpórea de $145 \mathrm{~min}$ (variando de 81-220 $\mathrm{min}$ ).

Os dados foram obtidos de forma retrospectiva, através dos prontuários dos pacientes. Os pacientes foram acompanhados através de contatos com seu médico clínico geral e também através de questionário enviado. 
Gama H, Martin W, Naik S K - Remodelamento cirúrgico do coração no tratamento cirúrgico da cardiomiopatia isquêmica.

Rev Bras Cir Cardiovasc 2001; 16(2): 114-8.

\section{RESULTADOS}

Em oito dos pacientes foi usado balão intraaórtico no pré-operatório, indicado eletivamente. Dois pacientes necessitaram do balão intra-órtico no período pós-operatório. Vinte e três pacientes receberam drogas inotrópicas ainda na sala de operações, mantidas em média por 24 horas.

O tempo médio de ventilação foi de $31,7 \mathrm{~h}$ (variando de 7-240h) e o tempo médio de permanência na UTI foi de 65,7h (variando de 19-264h). Dois pacientes foram reintubados e necessitaram de ventilação devido à insuficiência respiratória e 2 foram reoperados por sangramento. Oito pacientes desenvolveram fibrilação atrial e 1 paciente teve acidente vascular cerebral. A mortalidade hospitalar foi de 22,3\% (6 pacientes), sendo que 4 faleceram por síndrome de baixo débito e 2 por sepse.

O acompanhamento médio foi de 20,7 meses (variando de 10-47 meses). Dezenove (70,3\%) pacientes permanecem vivos. Um paciente faleceu 3 meses após alta hospitalar e um outro faleceu 11 meses após. Não conseguimos estabelecer a causa mortis, uma vez que não foi realizada necropsia. Dos sobreviventes, 17 encontram-se em classes I e II da NYHA. Dois pacientes foram re-hospitalizados por angina pectoris, porém nenhum deles teve infarto do miocárdio documentado. Dois pacientes tiveram episódio de fibrilação atrial e 3 foram admitidos em hospitais distritais para tratamento de ICC, porém não houve necessidade de reinternação.

\section{COMENTÁRIOS}

A miocardiopatia de origem isquêmica pode apresentar, num período de 36 meses, uma mortalidade de $75 \%$ e mesmo em pacientes que não são suficientemente sintomáticos para transplante, essa mortalidade é superior a $70 \%$ (1). A mesma, constitui a indicação mais freqüente para transplante cardíaco, em nossa Instituição.

Devido à alta mortalidade de pacientes em lista de espera e a outras limitações, tal como, o número de doações de órgãos, é que decidimos reavaliar esses pacientes, visando oferecer uma alternativa paliativa através de técnica cirúrgica convencional.

A revascularização do miocárdio, em pacientes com função ventricular esquerda acentuadamente reduzida, pode melhorar os sintomas da doença isquêmica ${ }^{(1-3,6,7)}$.

A identificação de miocárdio hibernante é muito importante no sentido de ampliar a indicação de revascularização a estes pacientes (1-7). Entretanto, a dilatação do coração gera um aumento de stress na parede do VE o que pode explicar a deterioração destes pacientes ${ }^{(8)}$. BATISTA et al. ${ }^{(13)}$ descreveram a ventriculectomia parcial do VE, para pacientes portadores de miocardiopatia dilatada. $O$ princípio desta técnica é reduzir o volume do VE, restaurando a relação massa-volume, reduzindo assim o stress na parede ventricular diminuindo, portanto, o consumo de oxigênio e melhorando o desempenho do VE $(13,14)$. DOR et al. (16) descreveram o uso de um retalho intraventricular para o tratamento dos aneurismas de ventrículo esquerdo. O objetivo é reduzir o volume no final da diástole e portanto o stress na parede do VE (13). Recentemente, o uso desta técnica foi ampliado para áreas de acinesia, com melhora da função ventricular esquerda $(16,17)$. Utilizamos a técnica de Dor, uma vez que muito pouco se sabe da evolução da ventriculectomia parcial nos pacientes portadores de miocardiopatia isquêmica. Em pacientes que sofreram infarto do miocárdio, a ressecção de parte da parede livre do VE pode representar um grande comprometimento, uma vez que o septo interventricular pode não manter uma contração adequada. A utilização do retalho de Dacron implica na utilização de anticoagulante oral (warfarin) por 3 meses e aspirina por tempo indeterminado.

A regurgitação mitral na miocardiopatia isquêmica é corrigida, em sua maioria, apenas com anuloplastia, uma vez que a dilatação funcional do anel mitral é o achado mais freqüente. $O$ reparo da valva atua como um remodelamento, uma vez que provoca mudanças na geometria do ventrículo esquerdo $(21,22)$. Adicionalmente, corrige a regurgitação e reduz a impedância ao VE ${ }^{(20)}$, melhorando a função ventricular.

Utilizamos o anel rígido de Carpentier-Edwards que favorece a melhor coaptação das cúspides e remodelamento do anel no final da sístole (20). Embora o anel rígido restrinja a função dos músculos constritores da base do VE (24), não existe evidência clínica que demonstre diferença entre o anel de Carpentier e o de Duran, no tocante a função ventricular (25).

A mortalidade neste grupo de pacientes permanece alta, porém algumas estratégias foram introduzidas no manuseio destes pacientes, tais como: a utilização de balão intra-aórtico no préoperatório, no sentido de dar mais estabilidade ao paciente, durante a indução anestésica e melhorar os resultados pós-operatórios (26,27); utilização de nutrição parenteral no pré-operatório (28); administração imediata, no pós-operatório de inibidores da enzima de conversão e antiarrítmicos quando necessário.

Mesmo quando bem indicados, estes pacientes são de alto risco e de difícil manuseio. A decisão 
Gama H, Martin W, Naik S K - Remodelamento cirúrgico do coração no tratamento cirúrgico da cardiomiopatia isquêmica. Rev Bras Cir Cardiovasc 2001; 16(2): 114-8.

cirúrgica é baseada na interpretação dos achados ecocardiográficos e angiográficos. Essa interpretação é puramente qualitativa. É um procedimento paliativo que pode ser usado em pacientes que apresentam alguma contra-indicação para transplante ou em pacientes que são enviados para transplante, mas que ainda podem obter benefício com operação convencional.

Necessitamos um grupo maior de pacientes e um acompanhamento mais longo para avaliar a sua evolução a longo prazo e maior investigação para avaliar o impacto desta operação na geometria do ventrículo esquerdo, bem como a melhoria em sua função, além de avaliar a função da valva mitral. Dos 19 pacientes que sobreviveram, apenas 3 concordaram em retornar ao hospital para realização de ecocardiograma e de cintilografia cardíaca, porém ainda não tiveram os exames realizados.

CONCLUSÃO

O remodelamento cirúrgico do coração é um procedimento paliativo que pode ser usado em pacientes portadores de miocardiopatia isquêmica, que apresentam alguma contraindicação para o transplante, mas que ainda podem obter benefício com operação convencional. Apresenta alta mortalidade hospitalar, porém morbidade aceitável e bom resultado imediato e a médio prazo.

Gama H, Martin W, Naik S K - Cardiac surgical remodeling as a surgical treatment for ischemic cardiomyopathy. Rev Bras Cir Cardiovasc 2001; 16(2): 114-8.

ABSTRACT: Objective: We present a group of patients with end stage ischemic cardiomyophaty, operated with a combined procedure, involving myocardial revascularization, mitral valve anuloplasty and ventriculoplasty.

Material and Methods: Twenty seven patients, 22 males, whose mean age was 57.8 years. The majority were in NYHA classes III and IV and had a mean ejection fraction of $15 \%$. Eight patients had IABP inserted preoperatively. CPB, temperature $32^{\circ} \mathrm{C}$ and warm blood cardioplegia. Twenty-six received 74 grafts (3.1 per patient). 10 had Mitral anuloplasty and 4 Mitral and Tricuspid. Two cases had plication of the LV and 18 DOR procedure. The mean X-clamp time $59 \mathrm{~m}$ and CPB time $145 \mathrm{~m}$. Ten patients came off Bypass on IABP and 25 required inotropic support.

Results: There was no theatre mortality. Mean ventilation time $31.7 \mathrm{~h}$ and mean ICU time $65.7 \mathrm{~h}$. Three patients required reventilation, 2 reexploration for bleeding, 8 developed AF and 1 had a stroke. The mean hospital stay was 13 days and 6 patients $(22.2 \%)$ died in hospital. The main cause of death was low cardiac output syndrome. The minimal follow-up was 10 months and the maximum was 47 months (mean 20.7 months). Two patients died on this period. Nineteen patients were followed-up. Nineteen patients are alive (70.3\%). fifteen are in NYHA class I, 2 in class II and 2 in class III. None had MI. Five patients were readmitted. The reasons for admissions were in 2 cases for AF, 2 cases angina and in 3 patients CHF.

Conclusion: The cardiac surgical remodeling has high hospital mortality, but acceptable morbidity and reasonable hospital stay. The median term survival is also very acceptable giving the patients a better clinical status.

DESCRIPTORS: Cardiac surgical procedures, methods. Myocardial ischemia, surgical. 
Gama H, Martin W, Naik S K - Remodelamento cirúrgico do coração no tratamento cirúrgico da cardiomiopatia isquêmica.

Rev Bras Cir Cardiovasc 2001; 16(2): 114-8.

\section{REFERÊNCIAS BIBLIOGRÁFICAS}

1 Kron I L, Flanagan T L, Blackbourne L H, Schroeder R A, Nolan S P - Coronary revascularization rather than cardiac transplantation for chronic ischemic cardiomyopathy. Ann Surg 1989; 210: 348-54.

2 Blitz A \& Laks $\mathrm{H}$ - The role of coronary revascularization in the management of heart failure: identification of candidates and review of results. Curr Opin Cardiol 1996; 11: 276-90.

3 Kleikamp G, Posival H, Minami K, El Banayosy A, Korfer $\mathrm{R}$ - Ischemic cardiomyopathy-revascularization vs. transplantation. Eur J Cardiothorac Surg 1997; 11 (Suppl): S1-4.

4 Shivalkar B, Maes A, Borgers $\mathrm{M}$ et al. - Only hibernating myocardium invariably shows early recovery after coronary revascularization. Circulation 1996; 94: 308-15.

5 Wechsler A S \& Junod F L - Coronary bypass grafting in patients with chronic congestive heart failure. Circulation 1989; 79 (6 Pt 2): 192-6.

6 Beanlands R S, Hendry P J, Masters R G, de Kemp R A, Woodend K, Ruddy T D - Delay in revascularization is associated with increased mortality rate in patients with severe left ventricular dysfunction and viable myocardium on fluorine 18-fluorodeoxyglucose positron emission tomography imaging. Circulation 1998; 98 (19 Suppl): II51-6.

7 Langenburg S E, Buchanan S A, Blackbourne L H et al. - Predicting survival after coronary revascularization for ischemic cardiomyopathy. Ann Thorac Surg 1995; 60: $1193-7$

8 Hochberg M S, Parsonnet V, Gielchinsky I, Hussain S $M$ - Coronary artery bypass grafting in patients with ejection fractions below forty percent: early and late results in 466 patients. J Thorac Cardiovasc Surg 1983; 86: 519-27.

9 Tadesmir O, Kucukacksu S D, Vural K M, Katircioglu F $S$, Kutuk E, Bayazit K - A comparison of the early and the midterm results after dynamic cardiomyoplasty in patients with ischemic or idiopathic cardiomyopathy. $J$ Thorac Cardiovasc Surg 1997; 113: 173-81.

10 Bridges C R - Myocardial laser revascularization: the controversy and the data. Ann Thorac Surg 2000; 69: 655-62.

11 Sun B C, Catanese K A, Spanier T B et al. - 100 Longterm implantable left ventricular assist devices: the Columbia Presbyterian interim experience. Ann Thorac Surg 1999; 68: 688-94.

12 Kadner A, Chen R H, Adams D H - Heterotopic heart transplantation: experimental development and clinical experience. Eur J Cardiothorac Surg 2000; 17: 474-81.

13 Batista R J, Santos J L, Takeshita N, Bocchino L, Lima $P N$, Cunha MA - Partial left ventriculectomy to improve left ventricular function in end-stage heart disease. J Card Surg 1996; 11: 96-8.

14 Gorcsan $3^{\text {rd }} \mathrm{J}$, Feldman A M, Kormos R L, Mandarino W A, Demetris A J, Batista R J - Heterogeneous immediate effects of partial left ventriculectomy on cardiac performance. Circulation 1998; 97: 839-42.
15 Cox J L - Surgical management of left ventricular aneurysms: a clarification of the similarities and differences between the Jatene and Dor techniques. Semin Thorac Cardiovasc Surg 1997; 9: 131-8.

16 Dor V, Montiglio F, Sabatier M et al. - Left ventricular shape changes induced by aneurysmectomy with endoventricular circular patch plasty reconstruction. Eur Heart J 1994; 15: 1063-9.

17 Dor V, Sabatier M, Di Donato M, Maioli M, Toso A, Montiglio F - Late hemodynamic results after left ventricular patch repair associated with coronary grafting in patients with postinfarction akinetic or dyskinetic aneurysm of the left ventricle. $J$ Thorac Cardiovasc Surg 1995; 110: 1291-301.

18 Dor V - Reconstructive left ventricular surgery for postischemic akinetic dilatation. Semin Thorac Cardiovasc Surg 1997; 9: 139-45

19 Christenson J T, Simonet F, Maurice J, Bloch A, Velebit $\mathrm{V}$, Schmuziger M - Mitral regurgitation in patients with coronary artery disease and low left ventricular ejection fractions. How should it be treated? Texas Heart Institute J 1995; 22: 243-9.

20 Chen F Y, Adams D H, Aranki S F et al. - Mitral valve repair in cardiomyopathy. Circulation 1998; 98 (19 Suppl): II124-7.

21 Bolling S F, Pagani F D, Deeb G M, Bach D S Intermediate-term outcome of mitral reconstruction in cardiomyopathy. J Thorac Cardiovasc Surg 1998; 115: $381-8$

22 Bonchek L I, Olinger G N, Siegel R, Tresch D D, Keelan Jr. M H. - Left ventricular performance after mitral reconstruction for mitral regurgitation. $J$ Tthorac Cardiovasc Surg 1984; 88: 122-7.

23 Carpentier A, Deloche, A, Dauptain J et al. - A new reconstructive operation for correction of mitral and tricuspid insufficiency. J Thorac Cardiovasc Surg $1971 ; 61: 1-13$

24 van Rijk-Zwikker G L, Schipperheyn J J, Huysmans H A, Bruschke A V - Influence of mitral valve prosthesis or rigid mitral ring on left ventricular pump function: a study on exposed and isolated blood-perfused porcine hearts. Circulation 1989; 80(3 pt 1): 11-7.

25 Green G R, Dagum P, Glasson J R et al. - Semirigid or flexible mitral annuloplasty rings do not affect global or basal regional left ventricular systolic function. Circulation 1998; 98: II128-II136.

26 Christenson J T, Simonet F, Schmuziger M - The effect of preoperative intra-aortic balloon pump support in high risk patients requiring myocardial revascularization. $J$ Cardiovasc Surg (Torino) 1997; 38: 397-402.

27 Bregman D \& Kaskel P - Advances in percutaneous intraaortic balloon pumping. Criti Care Clin 1986; 2: 221-36.

28 Svedjeholm R, Hakanson E, Vanhanen I-Rationale for metabolic support with amino acids and glucoseinsulin-potassium (GIK) in cardiac surgery. Ann Thorac Surg 1995; 59(2 supl): S15-22. 